\title{
REMARKS ON QUASI-LINDELÖF SPACES
}

\author{
YAN-KUI SONG \\ (Received 15 December 2012; accepted 12 March 2013; first published online 12 June 2013)
}

\begin{abstract}
In this paper, we show that there exist a Tychonoff quasi-Lindelöf space $X$ and a compact space $Y$ such that $X \times Y$ is not quasi-Lindelöf. This answers negatively an open question of Petra Staynova.

2010 Mathematics subject classification: primary 54D20; secondary 54E18.

Keywords and phrases: Lindelöf, weakly Lindelöf, quasi-Lindelöf.
\end{abstract}

\section{Introduction}

By a space we mean a topological space. Let us recall that a space $X$ is Lindelöf if every open cover of $X$ has a countable subcover. As a generalisation of Lindelöfness, Frolik [3] defined a space $X$ to be weakly Lindelöf if for every open cover $\mathcal{U}$ of $X$ there exists a countable subset $\mathcal{V}$ of $\mathcal{U}$ such that $\overline{\cup\{V: V \in \mathcal{V}\}}=X$. Unfortunately, this property is not inherited by closed subspaces. Thus Arhangel'skiî [1] defined a space $X$ to be quasi-Lindelöf if every closed subspace of $X$ is weakly Lindelöf. Recently, Staynova [5, 6] studied the relationships between quasi-Lindelöf spaces and related spaces and investigated topological properties of quasi-Lindelöf spaces. In [7], Song and Zhang stated that the product of a weakly Lindelöf space and a compact space is weakly Lindelöf, for which a proof was provided by Staynova [5]. Thus Staynova $[5,6]$ asked the following question.

Problem 1.1. Is the product of a quasi-Lindelöf space and a compact space quasiLindelöf?

The purpose of this paper is to show that there exist a Tychonoff quasi-Lindelöf space $X$ and a compact space $Y$ such that $X \times Y$ is not quasi-Lindelöf, which gives a negative answer to the question.

Throughout this paper, the cardinality of a set $A$ is denoted by $|A|$. Let $\omega$ be the first infinite cardinal and $\mathrm{c}$ the cardinality of the set of all real numbers. As usual, a cardinal

The author acknowledges support from the National Natural Science Foundation (grant 11271036) of China.

(C) 2013 Australian Mathematical Publishing Association Inc. 0004-9727/2013 \$16.00 
is the initial ordinal and an ordinal is the set of smaller ordinals. Every cardinal is often viewed as a space with the usual order topology. Other terms and symbols that we do not define follow [2].

\section{Main result}

In the following, we give an example showing that the product of a Tychonoff quasiLindelöf space $X$ and a compact space $Y$ need not be quasi-Lindelöf. We need the following lemma from [5].

Leмma 2.1. If $X$ is a separable space, then $X$ is quasi-Lindelöf.

Example 2.2. There exist a Tychonoff quasi-Lindelöf space $X$ and a compact space $Y$ such that $X \times Y$ is not quasi-Lindelöf.

Proof. Let $\mathcal{R}$ be a maximal almost disjoint family of infinite subsets of $\omega$ with $|\mathcal{R}|=\mathfrak{c}$.

Let $X=\mathcal{R} \cup \omega$ be the Isbell-Mrówka space [4]. Then $X$ is quasi-Lindelöf by Lemma 2.1, since $\omega$ is a countable dense subset of $X$.

Let $D=\left\{d_{\alpha}: \alpha<\mathfrak{c}\right\}$ be the discrete space of cardinality $\mathfrak{c}$ and let $Y=D \cup\left\{d^{*}\right\}$ be the one-point compactification of $D$.

Now we show that $X \times Y$ is not quasi-Lindelöf. Since $|\mathcal{R}|=\mathfrak{c}$, we can enumerate $\mathcal{R}$ as $\left\{r_{\alpha}: \alpha<\mathfrak{c}\right\}$. Let $A=\left\{\left\langle r_{\alpha}, d_{\alpha}\right\rangle: \alpha<\mathfrak{c}\right\}$. Then $A$ is a closed subset of $X \times Y$ by the construction of the topology of $X \times Y$ with $|A|=\mathfrak{c}$. For each $\alpha<\mathfrak{c}$, let

$$
U_{\alpha}=X \times\left\{d_{\alpha}\right\}
$$

Then $U_{\alpha}$ is an open subset of $X \times Y$. Let $\mathcal{U}=\left\{U_{\alpha}: \alpha<\mathfrak{c}\right\}$. Then $\mathcal{U}$ is a family of open subsets of $X \times Y$ such that $A \subseteq \cup \mathcal{U}$. Let $\mathcal{V}$ be any finite or countably infinite subset of $\mathcal{U}$. It is not difficult to see that

$$
\bar{U}= \begin{cases}\cup \mathcal{V}, & \mathcal{V} \text { finite, } \\ \cup \mathcal{V} \cup\left(X \times\left\{d^{*}\right\},\right. & \mathcal{V} \text { infinite. }\end{cases}
$$

Let $\alpha_{0}=\sup \left\{\alpha: U_{\alpha} \in \mathcal{V}\right\}$. Then $\alpha_{0}<\mathfrak{c}$, since $\mathcal{V}$ is finite or countably infinite. If we pick $\alpha^{\prime}>\alpha_{0}$, then $U_{\alpha^{\prime}} \notin \mathcal{V}$. Thus

$$
\left\langle r_{\alpha^{\prime}}, d_{\alpha^{\prime}}\right\rangle \notin \overline{\bigcup \mathcal{V}}
$$

since $U_{\alpha^{\prime}}$ is the only element of $\mathcal{U}$ containing $\left\langle r_{\alpha^{\prime}}, d_{\alpha^{\prime}}\right\rangle$ and $\bar{\cup} \mathcal{V} \cap U_{\alpha^{\prime}}=\emptyset$, which completes the proof.

\section{Acknowledgements}

The author would like to thank Professor R. Li for his valuable suggestions. He would also like to thank the referee for valuable suggestions. 


\section{References}

[1] A. Arhangel'skiǔ, 'A theorem on cardinality', Russian Math. Surveys 34 (1979), 153-154.

[2] E. Engelking, General Topology, revised and completed edition. (Heldermann, Berlin, 1989).

[3] Z. Frolik, 'Generalizations of compact and Lindelöf spaces', Czechoslovak Math. J. 9(84) (1959), $172-217$.

[4] S. Mrówka, 'On completely regular spaces', Fund. Math. 41 (1954), 105-106.

[5] P. Staynova, 'A comparison of Lindelöf-type covering properties of topological spaces', RHIT Undergraduate Math. J. 12(2) (2011), 163-204.

[6] P. Staynova, 'A note on quasi-Lindelöf spaces'. arXiv:1212.2869v1.

[7] Y.-K. Song and Y.-Y. Zhang, 'Some remarks on almost Lindelöf spaces and weakly Lindelöf spaces', Mat. Vesnik. 62 (2010), 77-83.

YAN-KUI SONG, Institute of Mathematics, School of Mathematical Science, Nanjing Normal University, Nanjing 210023, PR China

e-mail: songyankui@njnu.edu.cn 